\title{
When does Convergence of Asset Price Processes Imply Convergence of Option Prices? *
}

\author{
Friedrich Hubalek Walter Schachermayer \\ Institut für Statistik, Universität Wien, \\ Brünnerstr.72, A-1210 Wien, Austria. \\ WWW http://ito.bwl.univie.ac.at/ ws chach \\ e-Mail fhubalek@stat1.bwl.univie.ac.at
}

\begin{abstract}
We consider weak convergence of a sequence of asset price models $\left(S^{n}\right)$ to a limiting asset price model $S$. A typical case for this situation is the convergence of a sequence of binomial models to the Black-Scholes model, as studied by Cox, Ross, and Rubinstein.

We put emphasis on two different aspects of this convergence: firstly we consider convergence with respect to the given "physical" probability measures $\left(P^{n}\right)$ and secondly with respect to the "risk-neutral" measures $\left(Q^{n}\right)$ for the asset price processes $\left(S^{n}\right)$. (In the case of non-uniqueness of the risk-neutral measures also the question of the "good choice" of $\left(Q^{n}\right)$ arises.) In particular we investigate under which conditions the weak convergence of $\left(P^{n}\right)$ to $P$ implies the weak convergence of $\left(Q^{n}\right)$ to $Q$ and thus the convergence of prices of derivative securities.

The main theorem of the present paper exhibits an intimate relation of this question with contiguity properties of the sequences of measures $\left(P^{n}\right)$ with respect to $\left(Q^{n}\right)$ which in turn is closely connected to asymptotic arbitrage properties of the sequence $\left(S^{n}\right)$ of security price processes.

We illustrate these results with general homogeneous binomial and some special trinomial models.
\end{abstract}

KEY WorDS: weak convergence, option prices, asymptotic arbitrage, contiguity, binomial models

\section{Introduction}

Since the seminal paper by Cox, Ross, and Rubinstein (1979) the approximation of continuous time asset price models by discrete time models is a well-known theme in Mathematical Finance. Rachev and Rüschendorf (1994) investigated in a systematic way the question which continuous time models may occur as limits of binomial models.

We shall address the following issue: there are two different aspects of weak convergence of a sequence $\left(S^{n}\right)$ of discrete time models which are relevant in the context of Mathematical Finance: firstly it is the usual question of convergence with respect to the original, sometimes

\footnotetext{
${ }^{*}$ This piece of research was supported by the Austrian Science Foundation (FWF) under grant SFB\#10 ('Adaptive Information Systems and Modelling in Economics and Management Science').
} 
called "physical", probability measures $\left(P^{n}\right)$; but secondly there is also the question of convergence with respect to the so-called "risk-neutral" measures $\left(Q^{n}\right)$, which, e.g., in the case of binomial models are unique (provided they exist). This sequence of probability measures is relevant for the convergence of the prices of derivative securities on the underlying stock price process $\left(S^{n}\right)$.

The general theme of this paper is: How is convergence of $\left(P^{n}\right)$ related to convergence of $\left(Q^{n}\right)$ ? More precisely: Under what conditions does the convergence of $\left(P^{n}\right)$ imply the convergence of $\left(Q^{n}\right)$ ?

Bearing in mind that the equivalent martingale measures $Q^{n}$ define (via taking discounted expectations) a pricing rule for derivatives (such as options) a rough reformulation of this question is formulated in the title of the present paper: When does convergence of asset price processes imply convergence of option prices?

Our aim is to make these intuitively formulated questions mathematically precise and to provide sufficient conditions for an affirmative answer. (We consider here convergence of processes in distribution, or equivalently with respect to weak convergence of the laws of the processes, exclusively. Related results and further references can be found in Jakubowski, Mémin, and Pagés (1989), Duffie and Protter (1991), Kurtz and Protter (1991), Cutland, Kopp, and Willinger (1993), Kurtz (1991), Madan, Milne, and Shefrin (1989), Nelson and Ramaswamy (1990), He (1990), Avram (1988), Mémin and Slominski (1991), Stricker (1984/85). An overview regarding various convergence concepts used in Mathematical Finance is Willinger and Taqqu (1991). The reader interested in pathwise approximation should also consult Eberlein (1991), Delbaen and Schachermayer (1996).)

The starting point is a puzzling and at first sight amazing example, which is due to Th. Schlumprecht and, independently, to K. Pötzelberger.

1.1 Example For $\mu \in \mathbb{R}, \sigma>0, \hat{\sigma}>0$ there is a sequence of binomial asset price models with discounted asset price processes $\left(S^{n}\right)$, physical probabilities $\left(P^{n}\right)$, and risk-neutral probabilities $\left(Q^{n}\right)$, such that

(i) the sequence $\left(S^{n} \mid P^{n}\right)$ converges weakly to $(S \mid P)$, which is geometric Brownian motion with parameters $\mu$ and $\sigma^{2}$,

(ii) the sequence $\left(S^{n} \mid Q^{n}\right)$ converges to $(S \mid Q)$, which is geometric Brownian motion with parameters $-\hat{\sigma}^{2} / 2$ and $\hat{\sigma}$.

As a consequence the (unique arbitrage-free) price of a European option on $S$ may be different (and, indeed, very different!) from the limit of the (unique arbitrage-free) prices of the same option on $S^{n}$.

The construction of such an example (see Section 3 below) is actually quite simple: the trick is to use a sequence of binomial models with different behavior for odd and even increments.

In order to analyze the phenomenon arising in this example let us recall the basic idea behind the entire theory of pricing derivatives by no-arbitrage-arguments: if a derivative is "wrongly priced" there should be a possibility for arbitrage. The situation described by Example 1.1 can loosely be described by saying that the option is "asymptotically wrongly priced". This leads to the idea that this "asymptotically wrong price" should be related to some kind of "asymptotic arbitrage".

The notion of asymptotic arbitrage was introduced (in several variants) and studied in the work of Kabanov and Kramkov (1994, 1998), Klein and Schachermayer (1996a, 1996b), and 
Klein (1996) and is intimately related to contiguity properties of the sequences of measures $\left(P^{n}\right)$ with respect to the sequence of measures $\left(Q^{n}\right)$ and vice versa.

It turns out that there is indeed a close connection along these lines:

1.2 Theorem Let $\left(S^{n} \mid P^{n}\right)$ be a sequence of (not necessarily complete) asset price models that converges weakly to the complete asset price model $(S \mid P)$.

Let $\left(Q^{n}\right)$ be a sequence of equivalent martingale measures for $\left(S^{n} \mid P^{n}\right)$, such that the sequence of terminal values $\left(S_{T}^{n}\right)$ is uniformly $\left(Q^{n}\right)$-integrable.

If the sequence $\left(Q^{n}\right)$ is contiguous with respect to $\left(P^{n}\right)$, then $\left(S^{n} \mid Q^{n}\right)$ converges weakly to $(S \mid Q)$, where $Q$ is the unique equivalent martingale measure for $(S \mid P)$.

Let us put the message of Theorem 1.2 (precise definitions of the used terms will be given below) into a more informal language: by assumption we fix, for each $\left(S^{n} \mid P^{n}\right)$, an equivalent martingale measure $Q^{n}$, which we consider (by taking expected values) as a pricing rule for derivatives. Note that we did not assume that each $Q^{n}$ is unique, i.e., that each $S^{n}$ under $P^{n}$ is a complete market; we only assume that the limiting model $S$ under $P$ is complete. Under a technical uniform integrability assumption the contiguity of $\left(Q^{n}\right)$ with respect to $\left(P^{n}\right)$ then implies the convergence of $\left(Q^{n}\right)$ to $Q$. In particular this implies convergence of prices of European options on $S^{n}$ to the prices of the corresponding European options on $S$. (We do not address convergence of American option prices here. Related questions and further references on American options can be found in Lamberton and Pagés (1990), Mulinacci and Pratelli (1996), Amin and Khanna (1994), Lamberton (1993).)

The contiguity of $\left(Q^{n}\right)$ with respect to $\left(P^{n}\right)$ is closely connected to the idea of asymptotic arbitrage: for example, if we make the additional assumption that each $Q^{n}$ is the unique equivalent martingale measure for $\left(S^{n} \mid P^{n}\right)$, e.g., in the case of binomial models, then $\left(Q^{n}\right)$ is contiguous with respect to $\left(P^{n}\right)$ iff there is no asymptotic arbitrage of second kind as was shown by Kabanov and Kramkov (1994) (compare also Klein and Schachermayer (1996a, 1996b), and Kabanov and Kramkov (1998) for related and more general results). Using this relation between contiguity and asymptotic arbitrage we obtain from Theorem 1.2:

1.3 Corollary In the setting of Theorem 1.2 suppose in addition that each $Q^{n}$ is the unique equivalent martingale measure for $\left(S^{n} \mid P^{n}\right)$.

If $\left(S^{n} \mid P^{n}\right)$ permits no asymptotic arbitrage of second kind then $\left(S^{n} \mid Q^{n}\right)$ weakly converges to $(S \mid Q)$.

The paper is organized as follows: in Section 2 we fix notation and definitions, and in Section 3 we do the construction of the "odd-even" Example 1.1.

In Section 4 we give the proof of Theorem 1.2. We also provide examples showing none of the assumptions can be dropped for the theorem to hold true. On the other hand, we also give an example showing that the reverse implication of the theorem does not hold true, i.e., convergence of $\left(S^{n} \mid Q^{n}\right)$ to $(S \mid Q)$ does not imply contiguity of $\left(Q^{n}\right)$ with respect to $\left(P^{n}\right)$, see however, Proposition 3.9 below for a partial reverse result.

On the other hand we show (Theorem 3.8) that for homogeneous binomial models (where, in particular, the distinction between the odd and even increments cannot be made) the phenomenon of Example 1.1 cannot occur: loosely speaking, in the case of homogeneous binomial models convergence of stock prices implies convergence of option prices. This result seems to be wellknown and of folklore type but we have been unable to trace a precise reference in the literature and so we provide a proof. 
In Section 5 we apply Theorem 1.2 to a homogeneous trinomial model (similar results can be obtained for more general block multinomial models). In this setting, for each $n \in \mathbb{N}$ the process $S^{n}$ does not define a complete market and there is a wide variety of possible choices of equivalent martingale measures $Q^{n}$; on the other hand, in our setting the limiting model $S$ is just geometric Brownian motion (with drift) and therefore defines a complete market. So we find ourselves precisely in the situation of Theorem 1.2.

For certain homogeneous trinomial models we give explicit necessary and sufficient conditions characterizing those sequences $\left(Q^{n}\right)$ of martingale measures such that $\left(S^{n} \mid Q^{n}\right)$ converges to $(S \mid Q)$.

\section{Acknowledgements}

We thank Thomas Schlumprecht for bringing to our attention Example 1.1 which was the starting point of this paper as well as Klaus Pötzelberger, who constructed independently this example and presented it in the Seminar on Stochastic Processes and Mathematical Statistics in the summer term 1997 in Vienna.

\section{Definitions and Notations}

2.1 Definition $A n$ asset price model is a filtered probability space $\left(\Omega, \mathcal{F},\left(\mathcal{F}_{t}\right)_{t \in[0, T]}, P\right)$ with an $\mathbb{R}^{d}$-valued semi-martingale $\left(S_{t}\right)_{t \in[0, T]}$ denoting the discounted price processes of $d$ assets.

A probability measure $Q$ on $\mathcal{F}$ will be called an equivalent martingale measure for the asset price model, if $Q$ is equivalent to $P$ and $S$ is a $Q$-martingale. If we do not specify the filtration the term martingale pertains to the (augmented, right-continuous) filtration generated by $S$. We shall sometimes write briefly $S$ for the asset price model, or $(S \mid P)$ and $(S \mid Q)$ if we want to stress that we consider $S$ relative to $P$ or $Q$ respectively.

We say that a sequence $\left(S^{n} \mid P^{n}\right)$ of asset price models converges weakly to an asset price model $(S \mid P)$, if the sequence of probability measures defined by $\left(S^{n} \mid P^{n}\right)$ on the space $D^{d}[0, T]$ of càdlàg trajectories equipped with the Skorokhod topology converges to the probability measure defined by $(S \mid P)$, with respect to the weak convergence of probability measures, cf. Jacod and Shiryaev (1987) or Billingsley (1968).

A remark on the above definition seems appropriate: usually an asset price model is defined as an $\mathbb{R}^{d+1}$-valued semimartingale $\left(S_{t}\right)_{t \in[0, T]}$, where the first coordinate $S_{0, t}$ plays the role of the bond or riskless asset, which is assumed to be a strictly positive process. The term equivalent martingale measure then pertains to a measure $Q \sim P$, under which the discounted processes $S_{1, t} / S_{0, t}, \ldots, S_{d, t} / S_{0, t}$ are martingales.

In the present paper we are not really interested in the convergence of the bond price processes, i.e., of the 0 -th coordinate $\left(S_{0}^{n}\right)$ of the asset price models; our interest rather focuses on the convergence of the $d$ stock-price processes. Mainly in order to simplify the notation we therefore consider from the very beginning the $d$-dimensional process $S=\left(S_{1}, \ldots, S_{d}\right)$ of discounted stock price processes; in other words we choose the popular approach to use the bond as numeraire (compare, e.g., Delbaen and Schachermayer 1995). It is the process of discounted stock prices which is relevant for the pricing of derivative securities and therefore this setting allows to give more compact formulations; we remark, however, that it is also possible - mutatis mutandis and involving more cumbersome formulations - to present our 
results in the language of $(d+1)$-dimensional processes taking also explicitly into account the convergence of the bond price processes.

To prove weak convergence in our examples we often use the following functional version of the Lindeberg-Lévy central limit theorem Jacod and Shiryaev (1987, VII.5.4). Without loss of generality we set the time horizon $T=1$.

2.2 Theorem Assume $\left(\xi_{k}^{n}\right)_{k=1, \ldots, n}$ is a rowwise independent triangular array under $\left(P^{n}\right)$ satisfying the condition

$$
\sum_{k=1}^{n} E_{P^{n}}\left[\left|\xi_{k}^{n}\right|^{2} 1_{\left\{\left|\xi_{k}^{n}\right|>\varepsilon\right\}}\right] \rightarrow 0
$$

for all $\varepsilon>0$ as $n \rightarrow \infty$. Let $X_{t}^{n}=\sum_{k=1}^{[n t]} \xi_{k}^{n}$ denote the partial sum process. If

$$
\sum_{k=1}^{[n t]} E_{P^{n}}\left[\xi_{k}^{n}\right] \rightarrow \mu t
$$

uniformly in $t \in[0,1]$ as $n \rightarrow \infty$ and

$$
\sum_{k=1}^{[n t]} V_{P^{n}}\left[\xi_{k}^{n}\right] \rightarrow \sigma^{2} t
$$

for any $t \in[0,1]$ as $n \rightarrow \infty$ then $\left(X \mid P^{n}\right) \rightarrow(X \mid P)$, which is Brownian motion with drift $\mu$ and variance $\sigma^{2}$ on $[0,1]$.

We also shall use the following lemma.

2.3 Lemma If $\left(X^{n} \mid P^{n}\right) \rightarrow(X \mid P)$ then $\left(\exp \left(X^{n}\right) \mid P^{n}\right) \rightarrow(\exp (X) \mid P)$.

This follows from the fact that exp is uniformly continuous on compact intervals, and therefore the mapping $\alpha \rightarrow \alpha \circ \exp$ is continuous from $D$ to $D$ Jacod and Shiryaev (1987, VI.1.15 and $3.8)$.

The subsequent notions of contiguity and entire separation can be found in Jacod and Shiryaev (1987), Witting and Müller-Funk (1995), Strasser (1985), Roussas (1972), Greenwood and Shiryaev (1985), Shiryaev (1984).

2.4 Definition (i) A sequence of probability measures $\left(Q^{n}\right)$ is contiguous to the sequence $\left(P^{n}\right)$, both defined on measure spaces $\left(\Omega^{n}, \mathcal{F}^{n}\right)$, if $P^{n}\left(A^{n}\right) \rightarrow 0$ implies $Q^{n}\left(A^{n}\right) \rightarrow 0$ as $n \rightarrow \infty$ for all $A^{n} \in \mathcal{F}^{n}$. We shall denote this by writing $\left(Q^{n}\right) \triangleleft\left(P^{n}\right)$. If $\left(Q^{n}\right) \triangleleft\left(P^{n}\right)$ and $\left(P^{n}\right) \triangleleft\left(Q^{n}\right)$ we say the sequences are mutually contiguous.

(ii) The sequences $\left(Q^{n}\right)$ and $\left(P^{n}\right)$ are entirely separated, if there is a subsequence $n_{k} \rightarrow \infty$ and for each $k$ a set $A^{n_{k}}$, such that $P^{n_{k}}\left(A^{n_{k}}\right) \rightarrow 1$ and $Q^{n_{k}}\left(A^{n_{k}}\right) \rightarrow 0$ as $k \rightarrow \infty$. We shall denote this by writing $\left(Q^{n}\right) \triangle\left(P^{n}\right)$.

A useful criterion for contiguity and entire separation is the following, which we adapt from Jacod and Shiryaev (1987, V.2.32) for our applications. 
2.5 Theorem Assume $\left(\xi_{k}^{n}\right)_{k=1, \ldots, n}$ is a row wise independent triangular array under $\left(P^{n}\right)$ as well as under $\left(Q^{n}\right)$. Let $p_{k}^{n}$ and $q_{k}^{n}$ denote the law of $\xi_{k}^{n}$ under $P^{n}$ resp. $Q^{n}$, and let

$$
h^{n}(\alpha)=\sum_{k=1}^{n}\left[1-H\left(\alpha ; p_{k}^{n}, q_{k}^{n}\right)\right],
$$

where $H\left(\alpha ; p_{k}^{n}, q_{k}^{n}\right)$ is the Hellinger integral of order $\alpha \in(0,1)$.

(i) We have $\left(Q^{n}\right) \triangleleft\left(P^{n}\right)$ iff

$$
\lim _{\alpha \rightarrow 0} \limsup _{n \rightarrow \infty} h^{n}(\alpha)=0 .
$$

(ii) We have $\left(Q^{n}\right) \triangle\left(P^{n}\right)$ iff there is $\alpha \in(0,1)$ such that

$$
\limsup _{n \rightarrow \infty} h^{n}(\alpha)=\infty \quad \text { or } \quad \liminf _{n \rightarrow \infty} \inf _{k=1, \ldots, n} H\left(\alpha ; p_{k}^{n}, q_{k}^{n}\right)=0,
$$

and in this case (2.6) holds for all $\alpha \in(0,1)$.

Note: We write $p_{k}^{n}$ and $q_{k}^{n}$ sometimes to denote probability distributions, sometimes to denote related probabilities, but we prefer this to introducing a further notation.

2.6 Definition (Harrison and Pliska (1981),Delbaen and Schachermayer (1994)) A predictable $\mathbb{R}^{d}$-valued process $H$ is called admissible for $S$, if the stochastic integral with respect to the process $S$, denoted by $(H \cdot S)_{t \in[0, T]}$ is well-defined and there is a constant $C>0$ such that $(H \cdot S)_{t} \geq-C$ for all $t \in[0, T]$.

2.7 Definition (Kabanov and Kramkov (1994, 1998))

(i) A sequence $\left(H^{n}\right)$ of admissible trading strategies realizes asymptotic arbitrage of first kind $\left(A A_{1}\right)$, if there are numbers $C^{n} \rightarrow \infty$ such that $\left(H^{n} \cdot S^{n}\right)_{t} \geq-1$, for $t \in[0, T]$, and $\limsup P^{n}\left[\left(H^{n} \cdot S^{n}\right)_{T} \geq C_{n}\right]>0$ as $n \rightarrow \infty$.

(ii) A sequence $\left(H^{n}\right)$ of admissible trading strategies realizes asymptotic arbitrage of the second kind $\left(A A_{2}\right)$, if there is a $c>0$, such that $\left(H^{n} \cdot S^{n}\right)_{t} \geq-1$, for $t \in[0, T]$, and $\limsup P^{n}\left[\left(H^{n} \cdot S^{n}\right)_{T} \geq c\right]=1$ as $n \rightarrow \infty$.

(iii) A sequence $\left(H^{n}\right)$ of admissible trading strategies realizes strong asymptotic arbitrage, if $\left(H^{n} \cdot S^{n}\right)_{t} \geq-1$, for $t \in[0, T]$, and limsup $P^{n}\left[\left(H^{n} \cdot S^{n}\right)_{T} \geq C\right]=1$ for any $C>0$ as $n \rightarrow \infty$.

If there are no subsequences permitting asymptotic arbitrage possibilities of first, second, or strong kind, we say there is no asymptotic arbitrage (NAA) of first, second, or strong kind, respectively.

To come to the last (formally) undefined concept appearing in Theorem 1.2: the uniform $Q^{n}$-integrability condition of the sequence $\left(S_{T}^{n}\right)$ with respect to the measures $\left(Q^{n}\right)$ means

$$
E_{Q^{n}}\left[\left|S_{T}^{n}\right| I_{\left\{\left|S_{T}^{n}\right|>C\right\}}\right] \rightarrow 0
$$

uniformly in $n \in \mathbb{N}$ as $C \rightarrow \infty$. This implies in the present context the uniform boundedness in $L^{1}\left(Q^{n}\right)$ and uniform integrability condition as defined in Meyer and Zheng (1984). This condition holds, for example, if $\left(S^{n}\right)$ is $L^{p}\left(Q^{n}\right)$-bounded for some $p>1$, i.e.,

$$
\sup _{n \in \mathbb{N}} E_{Q^{n}}\left[\left|S_{T}^{n}\right|^{p}\right]<\infty .
$$


2.1 Notation We write

$$
f=g+O(h), \quad \text { resp. } \quad f=g+\Theta(h),
$$

if there exists $C>0$ (resp. $c>0$ and $C>0$ ) such that

$$
|f-g| \leq C|h|, \quad \text { resp. } \quad c|h| \leq|f-g| \leq C|h|
$$

\section{The odd-even binomial model}

3.1 Definition A sequence of asset price models $\left(S^{n}\right)$ is called a binomial model if each discounted asset price process $\left(S^{n}\right)$ evolves as follows: For $t \in[0, T]$

$$
S_{t}^{n}=S_{0} \exp \left(\sum_{k=1}^{[n t]} \xi_{k}^{n}\right),
$$

where $S_{0}>0$ is a constant and the increments $\left(\xi_{k}^{n}\right)$ of the logarithmic discounted returns $X_{t}^{n}=\sum_{k=1}^{[n t]} \xi_{k}^{n}$ form a row wise independent triangular array. The random variables $\xi_{k}^{n}$ assume two values $U_{k}^{n}$ and $D_{k}^{n}$ with positive probabilities $p_{k}^{n}$ and $1-p_{k}^{n}$,

$$
P^{n}\left[\xi_{k}^{n}=U_{k}^{n}\right]=p_{k}^{n}, \quad P^{n}\left[\xi_{k}^{n}=D_{k}^{n}\right]=1-p_{k}^{n}
$$

for $k=1, \ldots, n$. To avoid trivial complications we always assume $D_{k}^{n}<0<U_{k}^{n}$.

The model is called homogeneous, if $\left(U_{k}^{n}, D_{k}^{n}, p_{k}^{n}\right)$ depend on $n$ but not on $k$. It is called an odd-even binomial model if these parameters depend only on $n$ and the parity of $k$.

For later usage we recall the following lemma.

3.2 Lemma A binomial model has a unique martingale measure $Q^{n}$, which can be characterized by the probabilities

$$
Q^{n}\left[\xi_{k}^{n}=U_{k}^{n}\right]=q_{k}^{n}, \quad Q^{n}\left[\xi_{k}^{n}=D_{k}^{n}\right]=1-q_{k}^{n},
$$

which are given by the familiar formula (Cox, Ross, and Rubinstein 1979; Rachev and Rüschendorf 1994; Shiryaev, Kabanov, Kramkov, and Mel'nikov 1994; Pliska 1997)

$$
q_{k}^{n}=\frac{1-e^{D_{k}^{n}}}{e^{U_{k}^{n}-e^{D_{k}^{n}}}}
$$

3.3 Definition An asset price model $S$ is called a Black-Scholes model with parameters $\left(\mu, \sigma^{2}\right)$ if the discounted asset price process $S$ evolves as follows: For $t \in[0, T]$

$$
S_{t}=S_{0} \exp \left(X_{t}\right)
$$

where $S_{0}>0$ and the logarithmic discounted returns satisfy $X_{t}=\left(\mu-\sigma^{2} / 2\right) t+\sigma W_{t}$ with a standard Brownian motion $W$. 
For the construction of Example 1.1 we define

$$
U_{2 j-1}^{n}=\frac{\sigma_{1}}{\sqrt{n}}+\frac{\mu}{n}, \quad D_{2 j-1}^{n}=-\frac{\sigma_{2}}{\sqrt{n}}+\frac{\mu}{n}, \quad p_{2 j-1}^{n}=p
$$

and

$$
U_{2 j}^{n}=\frac{\sigma_{2}}{\sqrt{n}}+\frac{\mu}{n}, \quad D_{2 j}^{n}=-\frac{\sigma_{1}}{\sqrt{n}}+\frac{\mu}{n}, \quad p_{2 j}^{n}=1-p
$$

for $j=1, \ldots,[n / 2]$, with $\sigma_{1}>0, \sigma_{2}>0$ and $0<p<1$ to be fixed later.

We claim that the discounted logarithmic returns $\left(X^{n} \mid P^{n}\right)$ converge in distribution to $(X \mid P)$, where the limit $X$ is under $P$ a Brownian motion with drift $\mu$ and volatility $\sigma=$ $\left(\sigma_{1}+\sigma_{2}\right) \sqrt{p(1-p)}$. Indeed, denoting by $E_{P^{n}}$ and $V_{P^{n}}$ expectation and variance with respect to $P^{n}$, an easy calculation shows that

$$
E_{P^{n}}\left[\xi_{k}^{n}\right]=(-1)^{k-1} \frac{p \sigma_{1}-(1-p) \sigma_{2}}{\sqrt{n}}+\frac{\mu}{n}, \quad V_{P^{n}}\left[\xi_{k}^{n}\right]=\frac{\left(\sigma_{1}+\sigma_{2}\right)^{2}}{n} p(1-p),
$$

for $k=1, \ldots, n$, therefore

$$
\sum_{k=1}^{[n t]} E_{P^{n}}\left[\xi_{t}^{n}\right] \rightarrow \mu t, \quad \sum_{k=1}^{[n t]} V_{P^{n}}\left[\xi_{t}^{n}\right] \rightarrow\left(\sigma_{1}+\sigma_{2}\right)^{2} p(1-p) t .
$$

Since $U_{k}^{n} \rightarrow 0$ and $D_{k}^{n} \rightarrow 0$ uniformly in $k$ as $n \rightarrow \infty$ our claim follows from the LindebergFeller central limit theorem 2.2 and 2.3. So far nothing very surprising.

For option pricing we are interested in the behavior of the above markets under the riskneutral probability measures. Inserting the special values of (3.6) and (3.7) into (3.4) we obtain asymptotically

$$
\begin{aligned}
& q_{2 j-1}^{n}=\frac{\sigma_{2}}{\sigma_{1}+\sigma_{2}}\left\{1-\left(\frac{\sigma_{1}}{2}+\frac{\mu}{\sigma_{2}}\right) \frac{1}{\sqrt{n}}+O\left(\frac{1}{n}\right)\right\}, \\
& q_{2 j}^{n}=\frac{\sigma_{1}}{\sigma_{1}+\sigma_{2}}\left\{1-\left(\frac{\sigma_{2}}{2}+\frac{\mu}{\sigma_{1}}\right) \frac{1}{\sqrt{n}}+O\left(\frac{1}{n}\right)\right\}
\end{aligned}
$$

uniformly in $j=1, \ldots,[n / 2]$ as $n \rightarrow \infty$. For the expectation and variance under $Q^{n}$, denoted by $E_{Q^{n}}$ and $V_{Q^{n}}$, we obtain

$$
E_{Q^{n}}\left[\xi_{k}^{n}\right]=-\frac{\sigma_{1} \sigma_{2}}{2} n^{-1}+O\left(n^{-3 / 2}\right), \quad V_{Q^{n}}\left[\xi_{k}^{n}\right]=\sigma_{1} \sigma_{2} n^{-1}+O\left(n^{-3 / 2}\right)
$$

for $k=1, \ldots, n$. Thus under $Q^{n}$

$$
\sum_{k=1}^{[n t]} E_{Q^{n}}\left[\xi_{t}^{n}\right] \rightarrow-\frac{\sigma_{1} \sigma_{2}}{2} t, \quad \sum_{k=1}^{[n t]} V_{Q^{n}}\left[\xi_{t}^{n}\right] \rightarrow \sigma_{1} \sigma_{2} t .
$$

Consequently the $\left(X^{n} \mid Q^{n}\right)$ converges to $(X \mid Q)$, where the limit $X$ under $Q$ is a Brownian motion with drift $-\sigma_{1} \sigma_{2} / 2$ and volatility $\hat{\sigma}=\sqrt{\sigma_{1} \sigma_{2}}$.

An elementary consideration shows that we can produce any combination of $\sigma>0$ and $\hat{\sigma}>0$ by choosing appropriate values for $\sigma_{1}>0, \sigma_{2}>0$ and $0<p<1$. This finishes the construction of Example 1.1. 
3.4 Remark If we consider the odd-even models as (controlled) Markov chain approximations to the limiting Brownian motion, then these models are not locally consistent in the sense of Kushner (1997), see also Kushner and Dupuis (1992).

Let us consider the consequences of Example 1.1 for option prices. A priori we could think of two ways to calculate the price of an option on $S$ : Either as limit of the prices of the corresponding option on $S^{n}$, or alternatively as discounted expectation under the martingale measure $Q$. We will show, that for the European call option any pair of values within the trivial bounds for arbitrage-free option prices may occur in this way.

3.5 Proposition For any $\mu \in \mathbb{R}, \sigma>0$ there is a sequence of odd-even binomial markets that converge under the original measures $P^{n}$ to a Black-Scholes market with parameters $\mu, \sigma^{2}$, but the price of a European call option with strike price $K \in \mathbb{R}$ approaches the lower arbitrage bound, i.e., under the risk-neutral measures $Q^{n}$

$$
E_{Q^{n}}\left[\left(S_{T}^{n}-K\right)^{+}\right] \rightarrow\left(S_{0}-K\right)^{+} .
$$

Also, for any $\mu \in \mathbb{R}, \sigma>0$ there is a sequence of odd-even binomial markets that converge under the original measures $P^{n}$ to a Black-Scholes market with parameters $\left(\mu, \sigma^{2}\right)$, but the price of a European call option with strike price $K \in \mathbb{R}$ approaches the upper arbitrage bound, i.e.

$$
E_{Q^{n}}\left[\left(S_{T}^{n}-K\right)^{+}\right] \rightarrow S_{0}
$$

Proof: We fix $\mu$ and $\sigma$ and choose an arbitrary $\hat{\sigma}>0$. We have seen that there is an odd-even model, such that the limit of $\left(S^{n} \mid P^{n}\right)$ and $\left(S^{n} \mid Q^{n}\right)$ are geometric Brownian motions with volatility $\sigma$ resp. $\hat{\sigma}$. A direct and wellknown calculation shows that, for fixed strike price $K$, the limit of the price of the European call option

$$
\lim _{n \rightarrow \infty} E_{Q^{n}}\left[\left(S_{1}^{n}-K\right)^{+}\right]=E_{Q}\left[\left(S_{1}-K\right)^{+}\right]=f(\hat{\sigma}),
$$

where $f(\hat{\sigma})$ denotes the price of the option in a Black-Scholes model with volatility $\hat{\sigma}$. Since

$$
\lim _{\hat{\sigma} \rightarrow 0} f(\hat{\sigma})=\left(S_{0}-K\right)^{+}, \quad \lim _{\hat{\sigma} \rightarrow \infty} f(\hat{\sigma})=S_{0},
$$

and $\hat{\sigma}>0$ was arbitrary any price within the trivial arbitrage bounds $\left(S_{0}-K\right)^{+}$and $S_{0}$ may occur as limiting option price of an odd-even binomial model, which approximates under the original measures a given Black-Scholes model.

In the next proposition we relate the "asymptotically wrong" option price, which arises if we choose $\sigma \neq \hat{\sigma}$ in Example 1.1 with the notion of asymptotic arbitrage.

3.6 Proposition If we have $\sigma \neq \hat{\sigma}$ in Example 1.1 above, then there are strong asymptotic arbitrage possibilities, and $\left(P^{n}\right) \triangle\left(Q^{n}\right)$.

Proof: Let $p_{k}^{n}$ and $q_{k}^{n}$ denote the distribution of $\xi_{k}^{n}$ under $P^{n}$ and $Q^{n}$ respectively. Then the Hellinger integral of order $1 / 2$ is given by

$$
H\left(p_{k}^{n}, q_{k}^{n}\right)=\sqrt{p_{k}^{n} q_{k}^{n}}+\sqrt{\left(1-p_{k}^{n}\right)\left(1-q_{k}^{n}\right)}
$$


For the odd-even model under discussion we get

$$
\lim _{n \rightarrow \infty} H\left(p_{k}^{n}, q_{k}^{n}\right)=\sqrt{p q}+\sqrt{(1-p)(1-q)}
$$

uniformly in $k=1, \ldots, n$ with $q=\sigma_{1} /\left(\sigma_{1}+\sigma_{2}\right)$. By assumption $\sigma \neq \hat{\sigma}$, so $p \neq q$, hence $1-\sqrt{p q}-\sqrt{(1-p)(1-q)}>0$. This implies

$$
h^{n}\left(\frac{1}{2}\right)=n\left[1-H\left(p_{k}^{n}, q_{k}^{n}\right)\right] \rightarrow \infty
$$

as $n \rightarrow \infty$. By 2.5 this is equivalent to entire separation, which is equivalent to strong asymptotic arbitrage Kabanov and Kramkov (1998, Prop.4). $\square$.

Actually this proof leads to an example, which was pointed out to us by K.Pötzelberger, that shows, that contiguity (or absence of asymptotic arbitrage) is not a necessary assumption for the conclusion of Theorem 1.2.

3.7 Example There is an odd-even model such that under the physical probability measures $\left(P^{n}\right)$ the sequence $\left(S^{n}\right)$ converges in distribution to geometric Brownian motion with parameters $\mu$ and $\sigma^{2}$, and under the risk neutral probability measures $\left(Q^{n}\right)$ the sequence of stock prices $\left(S^{n}\right)$ converges to the correct limit, i.e. geometric Brownian motion with parameters $-\sigma^{2} / 2$ and $\sigma$, although the sequence of binomial models permits strong asymptotic arbitrage.

Define an odd-even model as in Example 1.1 with $p \neq 1 / 2$ and $q=1-p$. Then the variance is not affected, and the limiting measures $P$ and $Q$ are equivalent, although by the 2.5 we have entire separation. $\square$.

In Rachev and Rüschendorf (1994, Theorem 2.2) necessary and sufficient conditions for convergence of a sequence of homogeneous binomial markets to a Black-Scholes market are given, as well as sufficient conditions for the convergence of option prices (see Rachev and Rüschendorf 1994, Theorem 3.1). First we demonstrate, that in the homogeneous situation convergence of the stock prices implies in fact convergence of option prices, or loosely speaking, 'homogeneous binomial models have automatically good convergence properties', $c f$. the discussion in Willinger and Taqqu $(1991,5.2)$. This theorem seems to be wellknown and of folklore type, it is implicit in Rachev and Rüschendorf (1994), it was mentioned to us by K. Pötzelberger, but we have been unable to trace a precise reference in the literature.

3.8 Theorem Suppose a sequence of homogeneous binomial models $\left(S^{n}\right)$ with $U^{n} \rightarrow 0$, $D^{n} \rightarrow 0$ converges in distribution under $P^{n}$ to a Black-Scholes model with parameters $\mu, \sigma^{2}$.Then under the corresponding martingale measures $\left(Q^{n}\right)$ the sequence $\left(S^{n} \mid Q^{n}\right)$ converges to the Black-Scholes model with parameters $-\sigma^{2} / 2, \sigma^{2}$.

Proof: Instead of applying Theorem 1.2 we prefer to give an elementary proof. The convergence assumption is equivalent to

$$
n\left[U^{n} p^{n}+D^{n}\left(1-p^{n}\right)\right] \rightarrow \mu, \quad n\left(U^{n}-D^{n}\right)^{2} p^{n}\left(1-p^{n}\right) \rightarrow \sigma^{2} .
$$

This follows from the central limit theorem, or may be deduced easily from the conditions given in Rachev and Rüschendorf (1994). We claim

$$
\sigma^{2}=-\lim n U^{n} D^{n} .
$$


By assumption $n\left(U^{n} p^{n}+D^{n}\left(1-p^{n}\right)\right) \rightarrow \mu$, so $U^{n} p^{n}+D^{n}\left(1-p^{n}\right)=O(1 / n)$. We can write

$$
\left(U^{n}-D^{n}\right) p^{n}=-D^{n}+O\left(\frac{1}{n}\right), \quad\left(U^{n}-D^{n}\right)\left(1-p^{n}\right)=U^{n}+O\left(\frac{1}{n}\right) .
$$

Multiplying these equations yields

$$
n\left(U^{n}-D^{n}\right)^{2} p^{n}\left(1-p^{n}\right)=-n D^{n} U^{n}+O\left(U^{n}\right)+O\left(D^{n}\right)+O\left(\frac{1}{n}\right) .
$$

We consider here only models with $U^{n} \rightarrow 0$ and $D^{n} \rightarrow 0$, so the claim is proved.

Now we calculate the asymptotic expansion of the risk neutral probabilities,

$$
q^{n}=\frac{-D^{n}}{U^{n}-D^{n}}\left\{1-\frac{U^{n}}{2}+O\left(U^{n}-D^{n}\right)^{2}\right\} .
$$

We find

$$
n\left(U^{n}-D^{n}\right)^{2} q^{n}\left(1-q^{n}\right)=-n D^{n} U^{n}+O\left(D^{n} U^{n}\left(U^{n}-D^{n}\right)\right)+O\left(\frac{U^{n}-D^{n}}{n}\right) .
$$

showing $n\left(U^{n}-D^{n}\right)^{2} q^{n}\left(1-q^{n}\right) \rightarrow \sigma^{2}$. Finally

$$
U^{n} q^{n}+D^{n}\left(1-q^{n}\right)=\frac{D^{n} U^{n}}{2}+O\left(U^{n}-D^{n}\right)^{3},
$$

showing $n\left(U^{n} q^{n}+D^{n}\left(1-q^{n}\right)\right) \rightarrow-\sigma^{2} / 2$.

In the setting of homogeneous binomial models we also can give a converse to Theorem 1.2.

3.9 Proposition Under the assumptions of Theorem 3.8 suppose that $\left(S^{n} \mid P^{n}\right)$ converges to a non degenerate limit, i.e. $\sigma>0$. Then we have no asymptotic arbitrage (neither of first or second kind) and therefore $\left(P^{n}\right)$ and $\left(Q^{n}\right)$ are mutually contiguous.

Proof: According to the $2.5\left(Q^{n}\right) \triangleleft\left(P^{n}\right)$ iff

$$
\lim _{\alpha \rightarrow 0} \limsup _{n \rightarrow \infty} \sum_{k=1}^{n}\left[1-H\left(\alpha ; \rho_{k}^{n}, \rho_{k}^{\prime n}\right)\right]=0
$$

In the homogeneous binomial world this equation becomes

$$
\lim _{\alpha \rightarrow 0} \limsup _{n \rightarrow \infty} n\left(1-p^{n \alpha} q^{n(1-\alpha)}-\left(1-p^{n}\right)^{\alpha}\left(1-q^{n}\right)^{(1-\alpha)}\right)=0 .
$$

From equation (3.20), applied to $P^{n}$ and $Q^{n}$, we know

$$
n\left(U^{n}-D^{n}\right)^{2} p^{n}\left(1-p^{n}\right) \rightarrow \sigma^{2}, \quad n\left(U^{n}-D^{n}\right)^{2} q^{n}\left(1-q^{n}\right) \rightarrow \sigma^{2},
$$

with $\sigma>0$, thus

$$
\frac{1}{U^{n}-D^{n}}=O\left(n^{1 / 2}\right), \quad \frac{p^{n}\left(1-p^{n}\right)}{q^{n}\left(1-q^{n}\right)} \rightarrow 1 .
$$


From equation (3.22), applied to $P^{n}$ and $Q^{n}$, we know

$$
p^{n}\left(U^{n}-D^{n}\right)=-D^{n}+O\left(\frac{1}{n}\right), \quad q^{n}\left(U^{n}-D^{n}\right)=-D^{n}+O\left(\frac{1}{n}\right) .
$$

Combining this estimate with (3.30) gives

$$
p^{n}-q^{n}=O\left(\frac{1}{n^{1 / 2}}\right) .
$$

Equations (3.32) and (3.30) imply $\lim p^{n} / q^{n}=1$, or equivalently

$$
q^{n}=p^{n}\left(1-\frac{w^{n}}{p^{n}}\right), \quad w^{n}=O\left(n^{-1 / 2}\right) .
$$

Plugging this expressions into (3.27) gives

$$
n\left(1-p^{n \alpha} q^{n(1-\alpha)}-\left(1-p^{n \alpha}\right)\left(1-q^{n}\right)^{(1-\alpha)}\right)=O(\alpha(1-\alpha)) .
$$

\section{Proof of the main theorem}

4.1 Proof of Theorem 1.2: To show convergence we use a method initiated by Prokhorov (see Jacod and Shiryaev 1987, VI.3.18): We prove that $\left(S^{n} \mid Q^{n}\right)$ is tight and that $(S \mid Q)$ is the only possible limit point.

We assumed that the sequence $\left(S^{n} \mid P^{n}\right)$ converges weakly, hence it is tight. Our contiguity assumption guarantees that $\left(S^{n} \mid Q^{n}\right)$ is tight as well (see Jacod and Shiryaev 1987, X.3.1).

We consider the models $\left(S^{n} \mid P^{n}\right)$ and $\left(S^{n} \mid Q^{n}\right)$ as probability measures on the space $D^{d}[0, T]$ of $\mathbb{R}^{d}$-valued càdlàg functions equipped with the Skorokhod topology.

Since $D^{d}[0, T]$ is a Polish space, for any weak accumulation point of $\left(S^{n} \mid Q^{n}\right)$, say $\left(S \mid Q^{\prime}\right)$, there is a subsequence $\left(n_{k}\right)_{k=1}^{\infty}$ with $\left(S^{n_{k}} \mid Q^{n_{k}}\right) \rightarrow\left(S \mid Q^{\prime}\right)$. The subsequence inherits uniform integrability.

We now are in a position to apply a theorem of Meyer-Zheng Meyer and Zheng (1984, Theorem 11), which asserts the following: if $\left(S^{n_{k}} \mid Q^{n_{k}}\right)$ is a sequence of martingales converging weakly to a process $\left(S \mid Q^{\prime}\right)$ with respect to the so called Meyer-Zheng topology on $D^{d}[0, T]$ (which is weaker than the Skorokhod topology) satisfying the uniform integrability condition given in the assumptions of Theorem 1.2, then the limit $\left(S \mid Q^{\prime}\right)$ again is a martingale (with respect to its natural filtration).

Hence we obtain that $Q^{\prime}$ is a martingale measure for $S$ and from our contiguity assumption we obtain that $Q^{\prime}$ is absolutely continuous with respect to $P$ (see Witting and Muiller-Funk $1995,6.113)$.

Using the easy Lemma 4.2 below we conclude that $Q^{\prime}$ equals the unique equivalent martingale measure $Q$ for $S$.

Hence $\left(S^{n} \mid Q^{n}\right)$ is a tight sequence with $(S \mid Q)$ being its unique weak accumulation point, which readily shows the weak convergence of $\left(S^{n} \mid Q^{n}\right)$ to $(S \mid Q)$.

4.2 Lemma Suppose $S$ is a $Q$-martingale and $Q$ is the only martingale measure equivalent to $P$. If $Q^{\prime}$ is a martingale measure for $S$, which is absolutely continuous with respect to $P$, then $Q=Q^{\prime}$. 
Proof: $Q^{\prime \prime}:=\frac{1}{2}\left(Q+Q^{\prime}\right)$ is also a martingale measure, and it is equivalent to $P$, thus $Q=Q^{\prime}$.

4.3 Remark Let us analyze the assumptions of Theorem 1.2 and convince ourselves that they indeed are necessary for the theorem to hold true. Firstly we deal with the assumption that $(S \mid P)$ is a complete arbitrage-free market, i.e., that there is a unique equivalent martingale measure $Q$ for $S$. Clearly this assumption cannot be dropped: indeed, if $(S \mid P)$ is such that the set $\mathcal{M}^{e}(S)$ of equivalent martingale measures consists of more than one element, we may choose $\left(S^{n} \mid P^{n}\right) \equiv(S \mid P)$, for all $n \in \mathbb{N}$, and may choose a sequence $Q^{n} \in \mathcal{M}^{e}\left(S^{n}\right)=\mathcal{M}^{e}(S)$ which verifies the assumptions of uniform integrability and contiguity and does not converge: for example, fix $Q^{\prime} \neq Q^{\prime \prime}$ in $\mathcal{M}^{e}(S)$ and let, for $j \in \mathbb{N}, Q^{2 j-1}=Q^{\prime}$ and $Q^{2 j}=Q^{\prime \prime}$.

This trivial example shows that in the context of non-complete limiting models $(S \mid P)$ the question has to be posed differently: we have to restrict ourselves to special elements $Q^{n}$ of $\mathcal{M}^{e}\left(S^{n}\right)$ and $\mathcal{M}^{e}(S)$, such as the minimal (Föllmer and Schweizer 1991), the variance optimal (Schweizer 1996; Delbaen and Schachermayer 1996), the Esscher measure (Gerber and Shiu 1994), the entropy minimizing measure (Frittelli 1996; Grandits 1998; Miyahara $1995)$ etc. and ask whether it is true that these special choices $Q^{n} \in \mathcal{M}^{e}\left(S^{n}\right)$ converge to the corresponding special choice $Q \in \mathcal{M}^{e}(S)$.

This question seems to be an interesting and challenging topic for future research. Let us mention in this context related results for the case of the minimal (Runggaldier and Schweizer 1995 ) and the variance-optimal martingale measure (Prigent 1995). A result on approximations of the variance-optimal martingale measure in $L^{2}$ is contained in Delbaen and Schachermayer (1996).

We now deal with the second technical assumption we had to impose in Theorem 1.2, the uniform $\left(Q^{n}\right)$-integrability of $\left(S^{n}\right)_{n=1}^{\infty}$. The subsequent Example 4.4 illustrates in the present context the well-known phenomenon that - in the absence of uniform integrability - the weak limit of a sequence of martingales need not to be a martingale (not even a local martingale).

4.4 Example Consider a odd-even model as above, with $\mu=0$ and this time

$$
U_{2 j-1}^{n}=\frac{\sigma}{\sqrt{n}}, \quad D_{2 j-1}^{n}=-\frac{\sigma}{\sqrt{n}},
$$

and

$$
U_{2 j}^{n}=\ln n, \quad D_{2 j}^{n}=-\frac{a}{n},
$$

where $j=1, \ldots,[n / 2]$ and $a>0$. For simplicity we set $R^{n}=0$. Choose the probabilities $P^{n}$ so, that $S^{n}$ is a $P^{n}$-martingale, i.e., such that (3.4) holds true, so that $P^{n}=Q^{n}$. An easy calculation shows

$$
\sum_{k=1}^{[n t]} E_{P^{n}}\left[\xi_{t}^{n}\right] \rightarrow-\left(\frac{\sigma^{2}}{4}+\frac{a}{2}\right) t, \quad \sum_{k=1}^{[n t]} V_{P^{n}}\left[\xi_{t}^{n}\right] \rightarrow \frac{\sigma^{2}}{2} t .
$$

Since $a>0$ the limiting measure $P$ is not a martingale measure (not even a local martingale measure), thus useless for the purpose of option pricing: it is not the (unique) martingale measure $Q$ associated to the limiting process $(S \mid P)$, which is geometric Brownian motion with parameters $-\sigma^{2} / 4+a / 2$ and $\sigma^{2} / 2$. 
Finally let us discuss the question whether there is a converse to Theorem 1.2, i.e., whether we can deduce from the convergence of $\left(S^{n} \mid Q^{n}\right)$ to $(S \mid Q)$ something about the contiguity of $\left(Q^{n}\right)_{n=1}^{\infty}$ with respect to $\left(P^{n}\right)_{n=1}^{\infty}$ ?

Unfortunately there is no hope for a general result in this direction (compare, however, Proposition 3.9 for a positive result in the case of homogeneous binomial models). It is a wellknown phenomenon in Mathematical Statistics (see, e.g., Strasser 1985; Witting and Miiller-Funk 1995) that in the case of weak convergence of $\left(S^{n} \mid P^{n}\right)$ to $(S \mid P)$ and $\left(S^{n} \mid Q^{n}\right)$ to $(S \mid Q)$ the absolute continuity of $Q$ with respect to $P$ does not imply the contiguity of $\left(Q^{n}\right)_{n=1}^{\infty}$ with respect to $\left(P^{n}\right)_{n=1}^{\infty}$. Example 3.7 illustrates this situation.

\section{Trinomial models}

In this section we shall consider homogeneous trinomial models. These are obvious extensions of the binomial models 3.1. The difference is that the increments $\xi_{k}^{n}$ of the logarithmic returns $X^{n}$ assume three values $U^{n}, M^{n}, D^{n}$ with positive probabilities. The resulting markets are incomplete. For simplicity we choose

$$
U^{n}=\frac{\sigma}{\sqrt{n}}, \quad M^{n}=0, \quad D^{n}=-\frac{\sigma}{\sqrt{n}},
$$

with some $\sigma>0$ and the probabilities

$$
p^{n}\left(U^{n}\right)=p^{n}\left(M^{n}\right)=p^{n}\left(D^{n}\right)=\frac{1}{3} .
$$

We will see, that in contrast to homogeneous binomial models (cf. Theorem 3.8) homogeneous trinomial models do not possess good convergence properties automatically.

5.1 Proposition (i) The sequence of trinomial asset price models $\left(S^{n} \mid P^{n}\right)$ defined above converges weakly to $(S \mid P)$, which is geometric Brownian motion with parameters $0,2 \sigma^{2} / 3$.

(ii) The family of equivalent martingale measures $Q^{n}$, under which the process is again a homogeneous trinomial model can be characterized by the probabilities

$$
q^{n}\left(U^{n}\right)=\lambda^{n} \frac{1-e^{D^{n}}}{e^{U^{n}}-e^{D^{n}}}, \quad q^{n}\left(M^{n}\right)=1-\lambda^{n}, \quad q^{n}\left(D^{n}\right)=\lambda^{n} \frac{e^{U^{n}}-1}{e^{U^{n}}-e^{D^{n}}}
$$

with $0<\lambda^{n}<1$.

(iii) If $\lambda^{n} \rightarrow 2 / 3$ as $n \rightarrow \infty$ then $\left(S^{n} \mid Q^{n}\right) \rightarrow(S \mid Q)$, which is geometric Brownian motion with parameters $-\sigma^{2} / 3,2 \sigma^{2} / 3$. So in this case $P$ is equivalent to $Q$. If

$$
\lambda^{n}=\frac{2}{3}+O\left(\frac{1}{\sqrt{n}}\right)
$$

then $\left(P^{n}\right)$ and $\left(Q^{n}\right)$ are mutually contiguous, otherwise we have entire separation.

Proof: (i) We have

$$
E_{P^{n}}\left[\xi_{k}^{n}\right]=0, \quad V_{P^{n}}\left[\xi_{k}^{n}\right]=\frac{2 \sigma^{2}}{3 n}
$$


and by Theorem $2.2\left(X^{n} \mid P^{n}\right) \rightarrow(X \mid P)$, where the limit $X$ is a Brownian motion with zero drift and variance $2 \sigma^{2} / 3$.

(ii) We are interested in martingale measures $Q^{n}$ preserving the independence of the increments. From the martingale equation $u=(k-1) / n$ and $t=k / n$ with $k=1, \ldots, n$

$$
E_{Q^{n}}\left[S_{t}^{n} \mid \mathcal{F}_{u}\right]=S_{u}^{n} E_{Q^{n}}\left[e^{\xi_{k}^{n}}\right]=S_{u}^{n}
$$

we see that these $\left(Q^{n}\right)$ can be characterized by

$$
e^{U^{n}} q^{n}\left(U^{n}\right)+1 \cdot q^{n}\left(M^{n}\right)+e^{D^{n}} q^{n}\left(D^{n}\right)=1 .
$$

The solutions (5.3) are convex combinations of the measure ignoring the increments with value zero and the measure assigning all mass to it. Then

$$
E_{Q^{n}}\left[\xi_{k}^{n}\right]=-\lambda^{n} \frac{\sigma^{2}}{2 n}+O\left(n^{-3 / 2}\right), \quad V_{Q^{n}}\left[\xi_{k}^{n}\right]=\lambda^{n} \frac{\sigma^{2}}{n}+O\left(n^{-3 / 2}\right) .
$$

If $\lambda^{n} \rightarrow \lambda$ by the Lindeberg-Feller theorem $\left(X^{n} \mid Q^{n}\right) \rightarrow(X \mid Q)$, where the limit is Brownian motion with drift $-\lambda \sigma^{2} / 3$ and variance $\lambda \sigma^{2}$. We have

$$
\lambda^{n} \rightarrow \frac{2}{3} \Longleftrightarrow \lim _{n \rightarrow \infty} V_{P^{n}}\left[X_{t}^{n}\right]=\lim _{n \rightarrow \infty} V_{Q^{n}}\left[X_{t}^{n}\right] \Longleftrightarrow P \sim Q
$$

(iii) To study contiguity with the criterion from Theorem 2.5 we must consider

$$
(5.10) h^{n}(\alpha)=n\left[1-p^{n}\left(U^{n}\right)^{\alpha} q^{n}\left(U^{n}\right)^{1-\alpha}-p^{n}\left(M^{n}\right)^{\alpha} q^{n}\left(M^{n}\right)^{1-\alpha}-p^{n}\left(D^{n}\right)^{\alpha} q^{n}\left(D^{n}\right)^{1-\alpha}\right] .
$$

First we will show that $\lambda^{n} \rightarrow 2 / 3$ is necessary for contiguity, next we refine the argument and get (5.4) as necessary conditions. Finally it turns out that this is actually sufficient for mutual contiguity.

$$
h^{n}\left(\frac{1}{2}\right)=n\left[1-\sqrt{\frac{1}{3} q^{n}\left(U^{n}\right)}-\sqrt{\frac{1}{3} q^{n}\left(M^{n}\right)}-\sqrt{\frac{1}{3} q^{n}\left(U^{n}\right)}\right]
$$

If we take any convergent subsequence $\left(\lambda^{n_{k}}\right)$ with limit $\lambda$ as $k \rightarrow \infty$, then

$$
q^{n_{k}}\left(U^{n_{k}}\right) \rightarrow \frac{\lambda}{2}, \quad q^{n_{k}}\left(M^{n_{k}}\right) \rightarrow 1-\lambda, \quad q^{n_{k}}\left(D^{n_{k}}\right) \rightarrow \frac{\lambda}{2},
$$

and

$$
\frac{h^{n}\left(\frac{1}{2}\right)}{n} \rightarrow 1-\sqrt{\frac{1}{6} \lambda}-\sqrt{\frac{1}{3}(1-\lambda)}-\sqrt{\frac{1}{6} \lambda} .
$$

If the sequence $\lambda^{n}$ does not converge we have

$$
\limsup _{n \rightarrow \infty} h^{n}\left(\frac{1}{2}\right)=\infty
$$

which means entire separation. From now on we assume $\lambda^{n}=\frac{2}{3}\left(1+\rho^{n}\right)$ with $\rho^{n} \rightarrow 0$. Using the asymptotic expression

$$
q^{n}=\frac{\lambda}{2}\left\{1-\frac{\sigma}{\sqrt{n}}+O\left(\frac{1}{n}\right)\right\}
$$


we obtain

$$
\frac{h^{n}\left(\frac{1}{2}\right)}{n}=1-\frac{2}{3} \sqrt{1+\rho^{n}}-\frac{1}{3} \sqrt{1-2 \rho^{n}}+O\left(\frac{1}{n}\right)=\Theta\left(\rho^{n}\right)^{2}+O\left(\frac{1}{n}\right) .
$$

Therefore $\limsup _{n \rightarrow \infty} h^{n}\left(\frac{1}{2}\right)<\infty$ implies $\rho^{n}=O\left(\frac{1}{\sqrt{n}}\right)$. With this estimate we can actually show

$$
h^{n}(\alpha)=n\left[1-\left(\frac{2}{3}\right)^{\alpha} \lambda^{n(1-\alpha)}-\left(\frac{1}{3}\right)^{\alpha}\left(1-\lambda^{n}\right)^{1-\alpha}\right]+O(\alpha(1-\alpha))
$$

uniformly in $\alpha \in(0,1)$. A Taylor expansion of $1-(2 / 3)^{\alpha} \lambda^{1-a}-(1 / 3)^{\alpha}(1-\lambda)^{1-\alpha}$ around $\lambda=2 / 3$ reveals

$$
1-\left(\frac{2}{3}\right)^{\alpha} \lambda^{1-\alpha}-\left(\frac{1}{3}\right)^{\alpha}(1-\lambda)^{1-\alpha}=\Theta\left(n \alpha(1-\alpha)\left(\lambda-\frac{2}{3}\right)^{2}\right),
$$

uniformly in $\lambda \in(0,1)$ and $\alpha \in(0,1)$. Theorem 2.5 implies mutual contiguity for $\lambda=$ $2 / 3+O\left(n^{-1 / 2}\right)$, entire separation otherwise.

\section{REFERENCES}

Amin, K., and A. Khanna (1994) : "Convergence of American Option Values from Discrete- to Continuous-Time Financial Models," Math. Finance, 4, 289-304.

Avram, F. (1988) : "Weak Convergence of the Variations, Iterated Integrals and Doleans-Dade Exponentials of Sequences of Semimartingales," The Annals of Probability, 16, 246-250.

Billingsley, P. (1968) : Convergence of Probability Measures, Wiley, New York.

Cox, J. C., S. A. Ross, and M. Rubinstein (1979) : "Option Pricing: A Simplified Approach," J. Financial Econ., 7, 229-264.

Cutland, N., E. Kopp, and W. Willinger (1993) : "From Discrete to Continuous Finanical Models: New Convergence Results for Option Pricing," Math. Finance, 3, 101-123.

Delbaen, F., and W. Schachermayer (1994) : "A General Version of the Fundamental Theorem of Asset Pricing," Mathematische Annalen, 300, 463-520.

Delbaen, F., and W. Schachermayer (1995) : "The No-Arbitrage Property Under a Change of Numéraire," Stochastics Stochastics Rep., 53, 213-226.

Delbaen, F., and W. Schachermayer (1996) : "The Variance-Optimal Martingale Measure for Continuous Processes," Bernoulli, 2(1), 81-105.

Duffie, D., and P. Protter (1991) : "From Discrete- to Continuous-Time Finance: Weak Convergence of the Financial Gain Process," Math. Finance, 2, 1-15.

Eberlein, E. (1991) : "On Modeling Questions in Security Valuation,” Math.Finance, $2,17-32$. 
Föllmer, H., and M. Schweizer: (1991): "Hedging of Contingent Claims under Incomplete Information," in Applied Stochastic Analysis, eds. M. H. A. Davis and R. J. Elliott, no. 5 in Stochastics Monographs, Gordon and Breach, London, 389414.

Frittelli, M.: (1996): "The Minimal Entropy Martingale Measure and the Valuation Problem in Incomplete Markets," Working paper \# 20, Dipartimento di Matematica, Universitá di Milano.

Gerber, H. U., and E. S. Shiu (1994) : "Option Pricing by Esscher Transforms," Trans. Soc. Actuar., XLVI, 98-140.

Grandits, P. (1998) : "The p-optimal Martingale Measure and its Asymptotic relation with the Minimal Entropy Martingale Measure," Bernoulli, (to appear).

Greenwood, P. E., and A. N. Shiryaev (1985) : Contiguity and the Statistical Invariance Principle, Philadelphia: Gordon and Breach.

Harrison, M., and S. Pliska (1981) : "Martingales and Stochastic Integrals in the Theory of Continuous Trading," Stoch. Process. Appl., 11, 215-260.

He, H. (1990) : "Convergence from Discrete- to Continuous-Time Contingent Claims Prices," Rev. Financial Stud., 3, 523-546.

JACOD, J., and A. Shinyaev (1987) : Limit Theorems for Stochastic Processes, Springer.

Jakubowski, A., J. Mémin, and G. Pagés (1989) : "Convergence en loi des suites d'intégrales stochastiques sur l'espace $\mathbb{D}^{1}$ de Skorokhod," Prob. Th. Rel. Fields, 81, 111-137.

Kabanov, Y., and D. Kramkov (1994) : "Large Financial Markets: Asymptotic Arbitrage and Contiguity," Theory Prob. Appl., 39, 222-228.

Kabanov, Y., and D. Kramkov (1998) : "Large Financial Markets: Asymptotic Arbitrage and Contiguity," Finance and Stochastics, (to appear).

Klein, I.: (1996): Asymptotic Arbitrage Theory, Doctoral thesis, Univ. Vienna.

Klein, I., and W. Schachermayer (1996a) : "Asymptotic Arbitrage in Non-Complete Large Financial Markets," Theory Prob. Appl., 41, 927-934.

Klein, I., and W. Schachermayer (1996b) : "A Quantitative and a Dual Version of the Halmos-Savage Theorem with Applications to Mathematical Finance," The Annals of Probability, 24, 867-881.

Kurtz, T. G. (1991) : "Random Time Changes and Convergence in Distribution Under the Meyer-Zheng Conditions," The Annals of Probability, 19, 1010-1034.

Kurtz, T. G., and P. Protter (1991) : "Weak Limit Theorems for Stochastic Integrals and Stochastic Differential Equations," The Annals of Probability, 19, 1035-1070.

Kushner, H. J.: (1997): "Numerical Methods for Stochastic Control Problems in Finance," in Mathematics of Derivative Securities, eds. M. A. H. Dempster and S. R. Pliska, no. 15 in Publications of the Newton Institute, Cambridge Univ. Press, 504527 .

Kushner, H. J., and P. G. Dupuis (1992) : Numerical Methods for Stochastic Control Problems in Continuous Time, New York: Springer-Verlag. 
Lamberton, D. (1993) : "Convergence of the Critical Price in the Approximation of American Options," Math. Finance, 3, 179-190.

Lamberton, D., and G. Pagés (1990) : "Sur l'approximation des réduites," Ann. Inst. H. Poincaré, 26, 331-355.

Madan, D. B., F. Milne, and H. Shefrin (1989) : "The Multinomial Option Pricing Model and Its Brownian and Poisson Limits," Rev. Financial Stud., 2, 251-265.

Mémin, J., and L. Slominski (1991) : "Condition UT et stabilité en loi des solutions d'équation différentielles stochastiques," Sem. Prob., XXV, 162-177, 1NM 1485.

Meyer, P. A., and W. A. Zheng (1984) : "Tightness Criteria for Laws of Semimartingales," Ann. Inst. H. Poincaré, 20.

Miyahara, Y.: (1995): "Canonical Measures and Entropy," in Proc. Seventh JapanRussia Symposium, eds. S. Watanabe, M. Fukushima, Y. Prohorov, and A. Shiryaev, no. 17 in Probability Theory and Mathematical Statistics, World Scientific, Tokyo.

Mulinacci, S., and M. Pratelli: (1996): "Functional Convergence of Snell Envelopes; Applications to American Options Approximations." Preprint.

Nelson, D. B., and K. Ramaswamy (1990) : "Simple Binomial Processes as Diffusion Approximations in Financial Models," Rev. Financial Stud., 3, 393-430.

Pliska, S. R. (1997) : Introduction to Mathematical Finance: Discrete Time Models, Malden, Mass.: Blackwell.

Prigent, J.-L.: (1995): "Incomplete Markets: Convergence of options values under the minimal martingale measure," Tech. rep., THEMA. University of Cergy-Pontoise, France.

Rachev, S. T., and L. Rüschendorf (1994) : "Models for Option Prices," Theory Prob. Appl., 39(1), 120-152.

Roussas, G. G. (1972) : Contiguity of Probability Measures: Some Applications in Statistics, Cambridge Univ. Press.

Runggaldier, W. J., and M. Schweizer (1995) : "Convergence of Option Values under Incompleteness," Progress in Probability, 36, 365-384.

Schweizer, M. (1996) : "Approximation Pricing and the Variance-Optimal Martingale Measure," The Annals of Probability, 24, 206-236.

Shiryaev, A. (1984) : Probability, Springer, New York.

Shiryaev, A. N., Y. M. Kabanov, D. O. Kramkov, and A. V. Mel'nikov (1994): "Toward the Theory of Pricing of Options of Both European and American Types. I. Discrete Time," Theory Prob. Appl., 39(1), 14-60.

Strasser, H. (1985) : Mathematical Theory of Statistics, Walter de Gruyter, Berlin.

Stricker, C. (1984/85) : "Loi de semimartingales et critères de compacité," Sem. Prob., XIX, 209-217, LNM 1123.

Willinger, W., and M. S. Taqqu (1991) : "Toward a Convergence Theory for Continuous Stochastic Securities Market Models," Math. Finance, 1, 55-99.

Witting, H., and U. Müller-Funk (1995) : Mathematische Statistik II, B.G.Teubner, Stuttgart. 